\title{
Innate immunity and inflammation in NAFLD/NASH
}

\author{
Marco Arrese $^{1}$, Daniel Cabrera ${ }^{1,2}$, Alexis M. Kalergis ${ }^{3}$, and Ariel E. Feldstein ${ }^{4,}{ }^{*}$ \\ ${ }^{1}$ Departmento de Gastroenterología, Escuela de Medicina, Pontificia Universidad Católica de \\ Chile, Santiago, CHILE \\ 2Departamento de Ciencias Químicas y Biológicas, Facultad de Salud, Universidad Bernardo O \\ Higgins, Chile \\ ${ }^{3}$ Millenium Institute on Immunology and Immunotherapy, Departamento de Genética Molecular y \\ Microbiología; Facultad de Ciencias Biológicas, Pontificia Universidad Católica de Chile, \\ Santiago, Chile \\ ${ }^{4}$ Department of Pediatrics, University of California San Diego (UCSD), and Rady Children's \\ Hospital, San Diego, California,United States
}

\begin{abstract}
Inflammation and hepatocyte injury and death are the hallmarks of non-alcoholic steatohepatitis (NASH), the progressive form of non-alcoholic fatty liver disease (NAFLD), which is a currently burgeoning public health problem. Innate immune activation is a key factor in triggering and amplifying hepatic inflammation in NAFLD/NASH. Thus, identification of the underlying mechanisms by which immune cells in the liver recognize cell damage signals or the presence of pathogens or pathogen-derived factors that activate them is relevant from a therapeutic perspective. In this review, we present new insights into the factors promoting the inflammatory response in NASH including sterile cell death processes resulting from lipotoxicity in hepatocytes as well as into the altered gut-liver axis function, which involves translocation of bacterial products into portal circulation as a result of gut leakiness. We further delineate the key immune cell types involved and how they recognize both damage-associated molecular patterns (DAMPs) or pathogen-associated molecular patterns (PAMPs) through binding of surface-expressed pattern recognition receptors (PRRs), which initiate signaling cascades leading to injury amplification. The relevance of modulating these inflammatory signaling pathways as potential novel therapeutic strategies for the treatment of NASH is summarized.
\end{abstract}

\section{Keywords}

Liver disease; cell death; innate immunity; inflammation; therapy; NASH

Nonalcoholic fatty liver disease (NAFLD) is now one of the most common causes of chronic liver in both children and adults. Estimates of NAFLD prevalence in the general population

Corresponding author: Dr. Ariel E. Feldstein, Professor of Pediatrics, Chief, Division of Pediatric Gastroenterology, Hepatology, and Nutrition UCSD, 3020 Children's Way, MC 5030, San Diego, CA 92103-8450, Tel: (858) 966-8907, afeldstein@ucsd.edu.

Conflict of Interest: The authors state no conflict of interest. 
have been generated using a variety of non-invasive imaging techniques or measurement of liver enzymes and range between $20-40 \%$ in western countries, with the usual figure quoted at around $30 \%$ for adults and $10 \%$ for children [1,2]. NAFLD includes a wide spectrum of disorders associated with fat deposition in the liver that spans from isolated steatosis to nonalcoholic steatohepatitis (NASH) characterized by steatosis with hepatocellular injury, and inflammatory changes with or without fibrosis to NASH with advanced fibrosis and cirrhosis $[3,4]$. Natural history studies suggest that, although some patients with isolated steatosis may develop significant fibrosis, the majority of individuals in this group appear to have a benign non-progressive clinical course while those with NASH undisputedly may progress to cirrhosis [2,5]. Among the latter group of patients, those with liver fibrosis (stage 2 or higher) appear to be the ones at higher risk of overall and liver-related morbidity and mortality [6]. With NAFLD becoming increasingly common in the developed world over the last decade, NASH demonstrated the greatest increase as a cause of chronic liver disease among new liver transplant waiting list registrations, increasing almost twofold and becoming the second leading etiology of liver disease among new liver transplant waitlist registrations in 2013 [7,8].

In recent years, there has been tremendous progress in the uncovering of the mechanisms that contribute to development and progression of the disease. The disease involves a complex interaction between environmental factors, host genetics and gut microbiota and involves both intrahepatic and extrahepatic events [9-12]. In this Review, we focus on innate immune activation and inflammation two of the most characteristic features of NASH. We present new insights into the upstream triggers of inflammation in NASH including sterile cell death processes as a result of lipotoxicity in hepatocytes as well as into the altered gutliver axis function involving translocation of bacterial products into portal circulation as a result of a leaky gut. We further delineate the key cell types involved and the relevance of modulating the inflammatory signaling pathways as potential novel therapeutic strategies for the treatment of NASH.

\section{INFLAMMATION AND CELL DEATH IN NASH}

One of the key advances that help to explain the large spectrum of NAFLD seen in patients is the realization of the process called lipotoxicity [13-15]. Indeed, during the overloading of lipids in the liver, the types of fats that accumulate and the way that liver cells handle this lipid load may result in adaptation with the development of isolated hepatic steatosis or may trigger cell death by various distinct molecular mechanisms. The latter result in the release of stressed signals by hepatocytes, also called danger signals, triggering activation of sterile (i.e. in the absence of infection) inflammatory pathways that when perpetuated over time result in chronic injury and an abnormal wound healing response with fibrosis $[16,17]$.

Since the original description that caspase activation and TUNEL positive cells are a common pathological feature in the liver of NASH patients [18], a growth of data has demonstrated that hepatocyte cell death is a key process involved in NASH pathogenesis [12]. Sustained hepatocyte cell death has also been implicated in the development of hepatic fibrosis [17]. Also, to the classical modes of cell death, such as apoptosis and necrosis (oncosis), other forms of hepatic cell death have been more recently described in preclinical 
models and patients with NASH, including autophagic cell death, pyroptosis, and necroptosis [19-21]. Apoptosis, a highly organized and genetically controlled process, is the most investigated and best-defined form of programmed cell death in NASH and the predominant cell death pathway in this disease [12,22]. Apoptosis is initiated by either membrane receptors (extrinsic pathway) or intracellular stress leading to organelle dysfunction (intrinsic pathway). Both pathways tend to converge in the activation of effector caspases 3 and 7, which execute the final apoptotic changes [12]. Necrosis, or oncosis, is an accidental form of cell death with the fatal consequence being cellular oxygen deprivation whereby the generation of reactive oxygen species (ROS), leads to mitochondrial dysfunction and a drop in ATP levels below the threshold required to maintain cellular integrity [19]. The latter induces membrane rupture with the release of cellular contents. While ROS production and mitochondrial dysfunction are a central feature of NASH, necrotic cell death is a rare histopathological feature of the disease. Necroptosis is induced by the same death receptors that activate the extrinsic apoptotic pathway, namely Tumor Necrosis Factor Receptor-1 (TNF-R1), and Fas [21]. Upon interaction of receptor protein kinases 1 and 3 (RIP1 and RIP3), and a deficiency or absence of caspase 8, cell death that morphologically resembles necrosis occurs [23]. Controversy exists on the potential role of this form of cell death in NASH [12]. Pyroptosis is a novel caspase-1 dependent form of programmed cell death that has been recently shown to occur in vivo during liver injury and that shares features of apoptosis such as DNA fragmentation and necrosis such as plasma membrane permeabilization [20]. Pyroptosis results in the formation of discretely sized ionpermeable pores in the plasma membrane, which leads to water influx and cell swelling. While growing evidence points to an important role for NLRP3 Inflammasome in inflammation and fibrosis in NASH [24,25], the role of pyroptosis in this disease has yet to be explored.

Stressed or dying hepatocytes during lipotoxicity are capable of releasing intracellular molecules called damage-associated molecular patterns or DAMPs that can act on various immune cells in the liver (discussed below) to initiate a homeostatic, wound healing response to repair tissue injury $[17,19]$. However, the persistence of these signals can induce an exuberant response that can result in a full inflammatory response with tissue inflammation and excessive scarring leading to advanced fibrosis and ultimately to cirrhosis.

\section{ROLE OF INNATE IMMUNITY IN NASH}

In recent years, the role of the innate immune response in NAFLD has been focus of intense research. Activation and recruitment of immune cells in the liver either by local signals or signals derived from the adipose tissue or the gut, related to changes in microbial balance and/or bacterial translocation, may promote the inflammatory response leading to cell injury and death, thus promoting NAFLD disease progression. In particular, the role of intracellular or surface-expressed pattern recognition receptors (PRRs), which are critical in recognizing cell damage and pathogen invasion, as relevant players in NAFLD/NASH, is being unveiled [26]. In the following paragraphs, current information on these topics is reviewed focusing mainly on liver phenomena. Due to space restrictions, we will not discuss the role of adaptive immune mechanisms in NAFLD/NASH, which has been recently reviewed elsewhere [26,27]. 


\section{Damage-Associated Molecular Patterns (DAMPS), pathogen-associated molecular patterns (PAMPs) and its receptors}

Sterile inflammation occurs in the absence of pathogens or external antigens and is an important mechanism of liver injury in liver diseases [28] particularly in NASH where it probably contributes to ongoing inflammation and disease progression $[29,30]$. The acronym DAMPs refers to a set of intracellular molecules that are released or secreted upon the occurrence of cellular injury or death [31,32] and seem to be key inducers of sterile inflammation. A number of diverse molecules have been recognized as DAMPs including nuclear factors such as the high-mobility group box 1 [HMGB1], nuclear and mitochondrial DNA, purine nucleotides (i.e., ATP, UTP) and uric acid among others although its relative contribution to inflammation in the setting of liver disease is ill defined [28,33]. In addition to DAMPs, PAMPs are also at play in determining liver injury in NAFLD/NASH. PAMPs refer to a number of bacterial products including bacterial lipopolysaccharide (LPS), that derives from the cellular wall of gram-negative bacteria, and other molecules such as peptidoglycans, bacterial lipoproteins flagellins, bacterial RNA and DNA and others that can reach the liver upon disruption of the intestinal mucosal barrier (see below) and locally activate innate immune cells triggering intracellular signaling cascades that amplify injury.

DAMPs and PAMPs bind the PRRs, triggering a local inflammatory response and creating an injury amplification loop leading to organ damage (Figure 1). This is essentially mediated by the production of inflammatory cytokines such as Tumor necrosis factor-alpha (TNF-a) and Interleukin-6 (IL-6), which, in addition to promoting inflammation, have important metabolic effects influencing insulin resistance and lipid metabolism [9,11]. Among PRRs, Toll-like receptors (TLRs) are the best characterized and comprise a family of cell surface and endocytic receptors that are expressed in most liver cells including hepatocytes, Kupffer cells (KCs), hepatic stellate cells (HSCs), biliary epithelial cells and sinusoidal endothelial cells with each cell population exhibiting a different pattern of TLR expression [34-36]. The most studied TLRs in the liver, and particularly in NASH, are the TLR2, TLR4, and TLR9 [35-37]. These receptors recognize specific invariant motifs presents in pathogen molecules. While TLR2 recognizes peptidoglycans, TLR4 and TLR9 recognize bacterial LPS and DNA respectively $[38,39]$.

The role of TLRs in NAFLD/NASH has been studied using genetic ablation of these proteins in mice (reviewed in [37]). Suppression of TLR4, the receptor for LPS, and TLR-9, that binds DNA derived from intestinal bacteria, results in a marked attenuation of the inflammatory phenotype in different experimental models of NASH [36,40]. The role of TLR2, which recognizes some components of Gram-positive bacterial cell walls, is more controversial and discrepant data has been published [40,41].

The nucleotide oligomerization domain (NOD)-like receptors (NLRs) also belong to the PRR family and can recognize DAMPs and PAMPs. Activation of NLRs promotes the assembly of inflammasome proteins leading to cell death through the activation of caspase-1 and production of mature forms of IL-1 and IL-18 [42]. The role of inflammasome activation in NAFLD/NASH has received significant attention in recent years [25]. Work from one of the author's laboratory $[20,43]$ has demonstrated that NLRP3 inflammasome activation is associated to hepatocyte pyroptosis, an inflammasome-dependent cell death 
process, and that lack of this receptor attenuates inflammation and fibrosis in experimental NASH underscoring the relevance of this pathway.

\section{Cellular players}

Kupffer cells (KCs): are the resident macrophages of the liver located in the hepatic sinusoids, in the portal tract, and hepatic lymph nodes [44]. This cell type derives from circulating monocytes and represents around $15 \%$ of the liver cells being the largest tissuespecific reservoir of macrophages in the body [45]. In a healthy liver, the major immune function of $\mathrm{KCs}$ is to phagocyte pathogens or bacterial-derived products coming from portal vein circulation, constituting the final barrier to prevent spreading of these products to the peripheral circulation. KCs also phagocyte cell debris of neighboring cells and present antigens to cytotoxic and regulatory $\mathrm{T}$ cells [46].

Activation of KCs plays a key role in NAFLD pathogenesis and progression as demonstrated by studies showing that depletion of these cells attenuates insulin resistance, inflammatory development, and even fibrosis [46]. Expansion of $\mathrm{KC}$ is an early phenomenon in NASH and precedes the recruitment of other immune cells [47].

As other macrophages, KCs can undergo several forms of activation depending on the local environment [44]. Thus, KCs exhibit a range of polarized forms that has been classified in two broad phenotypes, the classically activated M1 macrophages, and the alternative M2 phenotype. While M1phenotype is regarded as essentially pro-inflammatory, M2 is considered "immunoregulatory" due to its involvement in wound healing and antiinflammation as well as, in some circumstances, pro-inflammation. However, there is ample agreement that this dichotomous concept does not entirely reflect the intricate biology of macrophage subsets as in some conditions KCs may simultaneously express markers of M1 and M2 differentiation [48].

The main activators that drive the classic "M1" phenotype of KCs are PAMPs including LPS and other gut-derived bacterial products that bind to the TLRs in KCs resulting in the production of pro-inflammatory cytokines and chemokines such as TNF-a, IL-1 $\beta$, IL-12 and $\mathrm{C}-\mathrm{C}$ motif ligand 2 and 5 (CCL2 and CCL5). This is a key step to trigger local inflammation and to promote additional hepatocyte cell injury leading to the release of DAMPs. DAMPs in turn further activate KCs via TLRs-signaling pathways, thus creating a vicious inflammatory circle. Moreover, some of the above-mentioned cytokines (i.e. CCL2 and CCL5) have overlapping pro-inflammatory and pro-fibrotic properties, contributing to chemotaxis of inflammatory cells and to the activation of HSC [49], Figure 1. Of note, several studies have shown that, in the setting of NAFLD/NASH, KCs can be also be activated by lipid overload. This may involve different lipids such as free fatty acids, oxidized lipoproteins, ceramides diacylglycerols and free cholesterol that has been shown to accumulate intracellularly in KCs in experimental NAFLD/NASH determining overexpression of TLRs and an increased response to LPS [16,50]. This has also been observed in vitro using KCs treated with palmitic acid [51]. Thus, fat overload in KCs seems to be also relevant in promoting activation of these cells in NAFLD/NASH likely contributing to disease progression. 
In addition to M1 polarization form, the alternative $\mathrm{M} 2$ phenotype refers to $\mathrm{KCs}$ that secrete a distinct set of mediators including IL-13, IL-10, IL-4 and TGF- $a$ and that reciprocally interact with Th2 CD4 T-cells. M2 KCs are mainly involved in wound healing and resolution of inflammatory states $[44,48]$. However, the nature of these polarized KCs is far more complex, and in fact different subtypes (M2a, M2b, and M2c macrophages) has been recognized. These sub-types exhibit different functional activities and are induced by distinct regulators. Of note, another particular type of macropaghes called scar-associated macrophages (SAMs) has been described, which may facilitate the resolution of liver fibrosis due to the production of liver matrix metalloproteinase-13 [52]. The role of M2 polarization of KCs in NAFLD/NASH remains ill defined [44] although existing data showing that activation of the nuclear receptor PPAR- $\delta$ promote M2 activation and influences obesity-induced insulin resistance in mice suggest that this pathway could exploited to treat NAFLD/NASH [53].

Dendritic Cells-DCs are tolerogenic immune cells located around the central veins and portal tracts that collectively represent a small fraction of liver non-parenchymal cells. DCs are mainly originated in the bone marrow. DCs have may act as antigen-presenting cells as well as in apoptotic cell clearance and removal of necrotic debris, thus limiting sterile inflammation. The role of DCs in NASH is complex and controversial [54]. Indeed, available information on the role of DCs in NASH is scarce, and the techniques used to assess its role in liver injury have shortcomings [55]. It is thought that while immature and tolerogenic DCs predominate in physiological conditions, mature and pro-inflammatory DC population prevails during liver injury. In the setting of experimental NASH, Henning et al. [56] recently showed that DCs rapidly infiltrate the liver exhibiting an activated immune phenotype expressing increased levels of IL-6, TNF-a and MCP-1. Interestingly and somewhat counterintuitively, depletion of DCs in NASH livers led to worsening of hepatic inflammation and fibrosis suggesting that DCs can have and anti-inflammatory and antifibrotic role in the setting of NASH. Another recent study [57], suggest that some DCs in the inflamed liver may derive from monocytes expressing a combination of inflammatory monocyte and DC markers on their cell surface as well as the fractalkine receptor (CX3CR1). This DC subtype exhibits an increased production of inflammatory mediators and an efficient antigen-presenting function. The prevention of accumulation of these cells in experimental NASH reduced TNF-a levels and limited liver injury in mice [57] somewhat in contrast with previous observations by Henning et al. [56]. The latter underscore the need for better experimental tools, and particularly more specific surface markers for a better delineation of the role of DCs in NASH [58].

Neutrophils-Neutrophil accumulation is one of the main features of NASH, and it is thought that this cell type critically contributes to hepatocellular damage in this setting as they can exacerbate the ongoing inflammatory state by contributing to macrophage recruitment and through interaction with antigen-presenting cells. The release of myeloperoxidase, a pro-oxidant neutrophil enzyme, seems to be a relevant mechanism that enhances macrophage cytotoxicity and promotes inflammation and fibrosis in experimental models [59]. In fact, studies using myeloperoxidase-deficient mice have shown attenuation of NASH development and down-regulation of pro-inflammatory cytokine production in the 
liver [60]. Also, neutrophil-derived peptides may also enhance hepatic fibrogenesis in experimental NASH as recently shown by Ibusuki et al. [61]. Finally, existing data support a role of neutrophil in modulating hepatic insulin resistance in a mechanism that involves elastase, another neutrophil-derived protease, through promotion of the inflammatory response. This is supported by the observation that deletion of neutrophil elastase ameliorates liver inflammation in an NAFLD experimental dietary model of NASH [62].

Natural Killer (NK) and Natural Killer T cells (NKT)—NK cells are lymphoid cells that play a role in linking the innate and adaptive immune responses within the liver [63]. Interestingly, liver NK cells display different immunophenotypical and functional characteristics than those of peripheral NK cells; this is thought to be related to cross-talk with other liver cells. NK cell functions are tightly regulated by the stimulation of diverse activating and inhibitory surface receptors. Different studies have shown that NK cells may be activated in NASH in connection with elevated levels of several NK cell-activating cytokines (e.g., IL-12, Interferon-c and IL-18) and ligands [28,63]. However, there are discrepant data in this regard as obese subjects with NAFLD and rats fed with a diet deficient in methionine and choline (MCD), that induces NASH, exhibit decreased cytotoxic activity of NK cells [28]. Thus, further research is needed to establish precisely the role of NK in NAFLD/NASH.

NKT cells are unique immune cell subtype that expresses specific NK cells surface receptors as well as an antigen receptor (TCR) characteristic of conventional T cells. They mainly reside in the sinusoids providing intravascular immune surveillance [64]. Recent studies point to the existence of at least two NKT-cell subsets that can play opposing roles in sterile liver inflammation with type I NKT being pro-inflammatory and type II conferring protection against liver injury [65]. Interestingly, type I NKT cells are readily activated by lipids and, therefore, may play a role in NAFLD. Studies assessing this issue are difficult to interpret due to technical disparities, and results should be interpreted with caution. Interestingly, mice lacking NKT cells are more susceptible to develop fatty liver and gain more weight when fed with high-fat diet [66] and the leptin-deficient mice exhibit reduced liver steatosis and improved glucose homeostasis upon adoptive transfer of transfer of NKT cells [67]. These two studies suggest a role of NKT cell depletion in early NAFLD. Moreover, NKT depletion has been related to KCs activation and local IL-12 production as shown by Kremer et al. [68]. On the other hand, a study of liver samples from patients with different degrees of NASH injury showed that when the disease progressed to more severe stages, the liver becomes enriched in NKT cells [69], which is in agreement with a subsequent study showing accumulation of NKT cells in advanced disease [70]. Thus, in NAFLD/NASH NKT cells seem to be depleted during steatosis development but increase later in the course of the disease likely contributing to inflammation and fibrosis [71].

\section{ACTIVATION OF INNATE IMMUNITY IN NAFLD/NASH: THE GUT LIVER AXIS AND ADIPOSE TISSUE}

The concept of gut-liver axis [72-74] refers to the existence of a physiological crosstalk between the liver and intestine consisting of a myriad of signals evoking relevant 
immunological and metabolic effects in the target organ. In fact, bile secretion from the liver has important consequences beyond fat digestion. Secretion of biliary constituents to the intestinal lumen also has immune roles (secretion of $\operatorname{Ig} \mathrm{A}$ ) and metabolic effects (bile acids stimulate secretion of glucagon-like peptide 1 [GLP-1] and Fibroblast growth factor-19 [FGF19] in enterocytes, thus regulating insulin secretion and action as well as bile acid synthesis) [75]. Moreover, bile acids can also regulate intestinal microbiota and through that regulate intestinal barrier (IB) function [76]. The IB is a complex structure that consists of several complex protein-protein networks that mechanically bind adjacent enterocytes and seal the intercellular space, the mucus layer coating the cell surface and the Paneth cells [77]. Structures of the IB include desmosomes, adherens junctions, and tight junctions that are formed by transmembrane proteins extracellularly interacting with neighboring cells and intracellularly bound to adaptor proteins linked to the cytoskeleton. The main components of tight junctions are zonulin-1, claudin, and occludin [73]. The IB is critical in controlling the pass of certain food-derived or bacterial products into the portal circulation. In normal conditions and due to its strategic anatomical position, the liver plays a role in immune surveillance of the portal circulation clearing the low levels of gut-derived LPS and other bacterial products (i.e. bacterial DNA, peptidoglycans or other components of bacterial cell walls) physiologically present in the portal blood. Thus, if IB function is deranged, LPS and other bacterial-derived compounds increases in the portal blood leading to an increased activation of TLRs and other PRRs in the liver and triggering local inflammatory and fibrogenic responses. As mentioned earlier, activation of TLRs, particularly TLR-4 and TLR-9, by PAMPs seem to be a key phenomenon in liver injury amplification in NAFLD/ NASH $[35-37,72,74]$ as their signaling promotes disease progression in different experimental models. One issue that remains to be clarified is the potential existence of a hypersensitivity of NASH patients to LPS, which would explain inter-individual differences related to the extent of liver damage to a given endotoxemia level [74].

Alterations of IB in NAFLD/NASH have received a great deal of attention in recent years. Several studies have shown that intestinal permeability is altered in subjects with NAFLD and that those with NASH exhibit a stronger association with altered intestinal permeability suggesting that liver injury and inflammation may be modulated by the degree of disruption of gut barrier integrity $[72,78]$. A recent meta-analysis of the existing literature showed that nearly $40 \%$ of patients with NAFLD had evidence of increased intestinal permeability compared with $6.8 \%$ of healthy individuals [79]. Other studies have shown that obesity by itself, the commonest comorbidity of NAFLD, is associated with changes in IB function [80] and that major tight junction proteins are down-regulated in patients with NAFLD [81].

Although it is thought that alterations in intestinal tight junctions proteins, (i.e. zonula occludens- 1 and occludin) are the main molecular mechanisms contributing to IB dysfunction in NAFLD the underlying causes are not well understood. Experimental evidence suggests that certain dietary components can directly induce changes in tight junction proteins as has been shown in high-fat or high sucrose/high-fat diet fed mice [72,74]. Dietary factors can act either directly causing intestinal inflammation or inducing changes in intestinal microbiota determining microbial dysbiosis. Finally, a recent experimental paper suggests a role of the $\mathrm{G}$ protein-coupled chemokine receptor (CX3CR1) in controlling IB function [82]. This receptor is expressed in intestinal macrophages, which 
have high phagocytic capacity and limit translocation of PAMPs. CX3CR1 is also involved in the protection of intestinal epithelial cells through autocrine regulation of cell survival signals. Deletion of this receptor promotes portal endotoxemia and worsens steatohepatitis in mice due to IB dysfunction [82].

Signaling from a dysfunctional adipose tissue is also involved in hepatic innate immunity activation in NAFLD/NASH [83]. Insulin resistant adipose tissue undergoes uncontrolled lipolysis increasing serum free fatty acids available for ectopic tissue deposition. In the liver, as discussed earlier, fatty acids may directly contribute to KCs activation via direct TLR2/4 binding [84]. Also, dysfunctional adipose tissue also exhibits an imbalance in adipokine production consisting in a reduced production of adiponectin and increased production of leptin and resistin [83].

Adiponectin has potent anti-inflammatory effects via its influence on macrophage function leading to a reduction in TNF-a production by these cells [85]. Adiponectin may also promote polarization of KCs into the M2 phenotype stimulating the production of antiinflammatory cytokines such as interleukin-10 $[44,86]$. Thus, reduced serum adiponectin likely contributes to hepatic inflammation in NAFLD/NASH. On the other hand, increased leptin and resistin may directly promote pro-inflammatory responses on KCs. However, its contribution to NAFLD/NASH remains unclear.

\section{CONCLUSION AND PERSPECTIVES}

In summary, in recent years, both basic and clinical research have established that innate immune activation plays an important role in triggering and amplifying hepatic inflammation in NAFLD/NASH. Resident liver macrophages (KCs) along with other immune cells play a major role in recognizing both signals from injured or dying fat-laden hepatocytes and presence of bacterial-derived products, which determine its activation and a robust pro-inflammatory response. Bacterial products can reach the liver as a result of a disturbed IB integrity induced by dietary components or microbial dysbiosis, which have been recently found to be key factors in modulating liver injury in NAFLD/NASH. Finally, existing evidence suggests that signals arising from adipose tissue can also influence innate immune cells and modulate inflammation and fibrosis in the liver.

Indeed, current knowledge opens the possibility of designing new potential therapies or to understand and/or improve current treatments. Thus, Cenicriviroc, a dual C-C chemokine receptor (CCR) 2/5 antagonist that limits the recruitment of hepatic macrophages into the liver, is currently being tested in patients with NASH. CCR2 binds CCL2, which is relevant in both chemotaxis of KCs and activation of HSC in the liver contributing to inflammation and fibrosis in NAFLD/NASH. Cenicriviroc was first tested in experimental models and now in is being studied in a Phase 2 trial in NASH patients (https://clinicaltrials.gov/ct2/show/ NCT02217475). Also, other drugs being tested for NASH, such as obeticholic acid [87] and the dual PPARa/\& GFT-505 [88], partially act by limiting hepatic inflammation in the liver influencing bile acid metabolism, intestinal inflammation, and macrophage recruitment. Modulation of IB function may also be a potential therapeutic target that could be exploited after gaining more precise knowledge of how dietary components affects tight junctions 
proteins or through targeting changes in intestinal microbiota using prebiotics or probiotics. Of note, despite the existence of positive evidence from animal models [74], the clinical efficacy of probiotics/prebiotics in NAFLD/NASH remains to be demonstrated in large clinical trials. Also, pharmacological agents that are able of tightening tight-junctions improving IB dysfunction such as larazotide warrant future studies in this regard. Finally, adipokine-targeted interventions for the treatment of NAFLD/NASH remain an unmet need.

\section{Acknowledgments}

This work was funded by NIH grants R01 DK082451, U01 AA022489 to AEF, and by grants from the Fondo Nacional de Desarrollo Cient fico y Tecnologico (FONDECYT 1150327 to M.A. and FONDECYT PD3140396 to D.C.) and the Comision Nacional de Investigacion Cient fica y Tecnologica (grant PFB 12/2007, Basal Centre for Excellence in Science and Technology, M.A.) both from the Government of Chile. A.K. received support from the Millennium Institute on Immunology and Immunotherapy, Santiago Chile. We thank the assistance of Nicolas Triantafilo, MD in providing the figure for this article.

\section{Abbreviations used in this paper}

NAFLD

NASH

ROS

RIP1 and RIP3

TNF-R1

DAMPs

PRRs

PAMPs

HMGB1

LPS

TNF-a

IL

TLRs

KCs

HSC

DCs

NLRs

CCL2 and CCL5

PPAR- $\delta$
Nonalcoholic fatty liver disease

nonalcoholic steatohepatitis

reactive oxygen species

receptor protein kinases 1 and 3

Tumor Necrosis Factor Receptor-1

damage-associated molecular patterns

pattern recognition receptors

pathogen-associated molecular patterns

the high-mobility group box 1

lipopolysaccharide

Tumor necrosis factor-alpha

Interleukin

Toll-like receptors

Kupffer KCs

hepatic stellate cells

dendritic cells

nucleotide oligomerization NOD-like receptors domain

C-C motif ligand 2 and 5

peroxisome proliferator activator receptor delta 


$\begin{array}{ll}\text { CX3CR1 } & \text { fractalkine receptor } \\ \text { NK } & \text { Natural Killer } \\ \text { NKT } & \text { Natural Killer T } \\ \text { IB } & \text { intestinal barrier } \\ \text { GLP-1 } & \text { Glucagon-like peptide 1 } \\ \text { FGF19 } & \text { Fibroblast growth factor-19 } \\ \text { IL-4 } & \text { interleukin-4 } \\ \text { IFN- } \gamma & \text { interferon-gamma } \\ \text { PDGF } & \text { platelet-derived growth factor } \\ \text { NEU } & \text { Neutrophils } \\ \text { SAMs } & \text { Scar-associated Macrophages }\end{array}$

\section{References}

1. Sayiner M, Koenig A, Henry L, Younossi ZM. Epidemiology of Nonalcoholic Fatty Liver Disease and Nonalcoholic Steatohepatitis in the United States and the Rest of the World. Clinics in Liver Disease. Epub ahead of print.

2. Satapathy SK, Sanyal AJ. Epidemiology and Natural History of Nonalcoholic Fatty Liver Disease. Semin Liver Dis. 2015; 35:221-235. [PubMed: 26378640]

3. Yeh MM, Brunt EM. Pathological features of fatty liver disease. Gastroenterology. 2014; 147:754764. [PubMed: 25109884]

4. Burt AD, Lackner C, Tiniakos DG. Diagnosis and Assessment of NAFLD: Definitions and Histopathological Classification. Semin Liver Dis. 2015; 35:207-220. [PubMed: 26378639]

5. Marengo A, Jouness EI, Bugianesi E. Progression and Natural History of Nonalcoholic Fatty Liver Disease in Adults. Clinics in Liver Disease. Epub ahead of print.

6. Angulo P, Kleiner DE, Dam-Larsen S, et al. Liver Fibrosis, but No Other Histologic Features, Is Associated With Long-term Outcomes of Patients With Nonalcoholic Fatty Liver Disease. Gastroenterology. 2015; 149:389-397. [PubMed: 25935633]

7. Charlton MR, Burns JM, Pedersen RA, Watt KD, Heimbach JK, Dierkhising RA. Frequency and outcomes of liver transplantation for nonalcoholic steatohepatitis in the United States. Gastroenterology. 2011; 141:1249-1253. [PubMed: 21726509]

8. Darwish Murad S, Metselaar HJ. The invasion of fatty liver disease in liver transplantation. Transpl Int. 2015

9. Feldstein AE. Novel insights into the pathophysiology of nonalcoholic fatty liver disease. Semin Liver Dis. 2010; 30:391-401. [PubMed: 20960378]

10. Higuera-de la Tijera F, Servin-Caamano AI. Pathophysiological mechanisms involved in nonalcoholic steatohepatitis and novel potential therapeutic targets. World J Hepatol. 2015; 7:12971301. [PubMed: 26052375]

11. Peverill W, Powell LW, Skoien R. Evolving concepts in the pathogenesis of NASH: beyond steatosis and inflammation. Int J Mol Sci. 2014; 15:8591-8638. [PubMed: 24830559]

12. Hirsova P, Gores GJ. Death Receptor-Mediated Cell Death and proinflammatory Signaling in Nonalcoholic Steatohepatitis. Cell Mol Gastroenterol Hepatol. 2015; 1:17-27. [PubMed: 25729762]

13. Trauner M, Arrese M, Wagner M. Fatty liver and lipotoxicity. Biochim Biophys Acta. 2010; 1801:299-310. [PubMed: 19857603] 
14. Ibrahim SH, Kohli R, Gores GJ. Mechanisms of lipotoxicity in NAFLD and clinical implications. J Pediatr Gastroenterol Nutr. 2011; 53:131-140. [PubMed: 21629127]

15. Zambo V, Simon-Szabo L, Szelenyi P, Kereszturi E, Banhegyi G, Csala M. Lipotoxicity in the liver. World J Hepatol. 2013; 5:550-557. [PubMed: 24179614]

16. Arguello G, Balboa E, Arrese M, Zanlungo S. Recent insights on the role of cholesterol in nonalcoholic fatty liver disease. Biochim Biophys Acta. 2015; 1852:1765-1778. [PubMed: 26027904]

17. Seki E, Schwabe RF. Hepatic inflammation and fibrosis: functional links and key pathways. Hepatology. 2015; 61:1066-1079. [PubMed: 25066777]

18. Feldstein AE, Canbay A, Angulo P, et al. Hepatocyte apoptosis and fas expression are prominent features of human nonalcoholic steatohepatitis. Gastroenterology. 2003; 125:437-443. [PubMed: 12891546]

19. Luedde T, Kaplowitz N, Schwabe RF. Cell death and cell death responses in liver disease: mechanisms and clinical relevance. Gastroenterology. 2014; 147:765-783. e764. [PubMed: 25046161]

20. Wree A, Eguchi A, McGeough MD, et al. NLRP3 inflammasome activation results in hepatocyte pyroptosis, liver inflammation, and fibrosis in mice. Hepatology. 2014; 59:898-910. [PubMed: 23813842]

21. Afonso MB, Rodrigues PM, Carvalho T, et al. Necroptosis is a key pathogenic event in human and experimental murine models of non-alcoholic steatohepatitis. Clin Sci (Lond). 2015; 129:721-739. [PubMed: 26201023]

22. Alkhouri N, Carter-Kent C, Feldstein AE. Apoptosis in nonalcoholic fatty liver disease: diagnostic and therapeutic implications. Expert Rev Gastroenterol Hepatol. 2011; 5:201-212. [PubMed: 21476915]

23. Moriwaki K, Chan FK. RIP3: a molecular switch for necrosis and inflammation. Genes Dev. 2013; 27:1640-1649. [PubMed: 23913919]

24. Mehal WZ. The inflammasome in liver injury and non-alcoholic fatty liver disease. Dig Dis. 2014; 32:507-515. [PubMed: 25034283]

25. Szabo G, Petrasek J. Inflammasome activation and function in liver disease. Nat Rev Gastroenterol Hepatol. 2015; 12:387-400. [PubMed: 26055245]

26. Sutti S, Bruzzi S, Albano E. The role of immune mechanisms in alcoholic and nonalcoholic steatohepatitis: a 2015 update. Expert Rev Gastroenterol Hepatol. 2015:1-11.

27. Jindal A, Bruzzi S, Sutti S, et al. Fat-laden macrophages modulate lobular inflammation in nonalcoholic steatohepatitis (NASH). Exp Mol Pathol. 2015; 99:155-162. [PubMed: 26112094]

28. Ganz M, Szabo G. Immune and inflammatory pathways in NASH. Hepatol Int. 2013; 7(Suppl 2): 771-781. [PubMed: 24587847]

29. Kubes P, Mehal WZ. Sterile inflammation in the liver. Gastroenterology. 2012; 143:1158-1172. [PubMed: 22982943]

30. Miura K, Yang L, van Rooijen N, Brenner DA, Ohnishi H, Seki E. Toll-like receptor 2 and palmitic acid cooperatively contribute to the development of nonalcoholic steatohepatitis through inflammasome activation in mice. Hepatology. 2013; 57:577-589. [PubMed: 22987396]

31. Garcia-Martinez I, Shaker ME, Mehal WZ. Therapeutic Opportunities in Damage-Associated Molecular Pattern-Driven Metabolic Diseases. Antioxidants \& redox signaling. 2015; 23:13051315. [PubMed: 26055926]

32. Uchida K. Natural antibodies as a sensor of electronegative damage-associated molecular patterns (DAMPs). Free Radical Biology \& Medicine. 2014; 72:156-161. [PubMed: 24657731]

33. Huebener P, Pradere JP, Hernandez C, et al. The HMGB1/RAGE axis triggers neutrophil-mediated injury amplification following necrosis. J Clin Invest. 2015; 125:539-550. [PubMed: 25562324]

34. Seki E, Brenner DA. Toll-like receptors and adaptor molecules in liver disease: update. Hepatology. 2008; 48:322-335. [PubMed: 18506843]

35. Kesar V, Odin JA. Toll-like receptors and liver disease. Liver Int. 2014; 34:184-196. [PubMed: 24118797]

36. Petrasek J, Csak T, Szabo G. Toll-like receptors in liver disease. Advances in Clinical Chemistry. 2013; 59:155-201. [PubMed: 23461136] 
37. Bieghs V, Trautwein C. Innate immune signaling and gut-liver interactions in non-alcoholic fatty liver disease. Hepatobiliary Surg Nutr. 2014; 3:377-385. [PubMed: 25568861]

38. Netea MG, van der Meer JW. Immunodeficiency and genetic defects of pattern-recognition receptors. The New England journal of medicine. 2011; 364:60-70. [PubMed: 21208109]

39. Takeuchi O, Akira S. Pattern recognition receptors and inflammation. Cell. 2010; 140:805-820. [PubMed: 20303872]

40. Miura K, Kodama Y, Inokuchi S, et al. Toll-like receptor 9 promotes steatohepatitis by induction of interleukin-1beta in mice. Gastroenterology. 2010; 139:323-334. e327. [PubMed: 20347818]

41. Ehses JA, Meier DT, Wueest S, et al. Toll-like receptor 2-deficient mice are protected from insulin resistance and beta cell dysfunction induced by a high-fat diet. Diabetologia. 2010; 53:1795-1806. [PubMed: 20407745]

42. Szabo G, Petrasek J. Inflammasome activation and function in liver disease. Nat Rev Gastroenterol Hepatol. 2015; 1247:387-400. [PubMed: 26055245]

43. Wree A, McGeough MD, Pena CA, et al. NLRP3 inflammasome activation is required for fibrosis development in NAFLD. J Mol Med (Berl). 2014; 92:1069-1082. [PubMed: 24861026]

44. Dixon LJ, Barnes M, Tang H, Pritchard MT, Nagy LE. Kupffer cells in the liver. Compr Physiol. 2013; 3:785-797. [PubMed: 23720329]

45. Duarte N, Coelho IC, Patarrao RS, Almeida JI, Penha-Goncalves C, Macedo MP. How Inflammation Impinges on NAFLD: A Role for Kupffer Cells. Biomed Res Int. 2015; 2015:984578. [PubMed: 26090470]

46. Lanthier N. Targeting Kupffer cells in non-alcoholic fatty liver disease/non-alcoholic steatohepatitis: Why and how? World J Hepatol. 2015; 7:2184-2188. [PubMed: 26380042]

47. Gadd VL, Skoien R, Powell EE, et al. The portal inflammatory infiltrate and ductular reaction in human nonalcoholic fatty liver disease. Hepatology. 2014; 59:1393-1405. [PubMed: 24254368]

48. Tacke F, Zimmermann HW. Macrophage heterogeneity in liver injury and fibrosis. J Hepatol. 2014; 60:1090-6. [PubMed: 24412603]

49. Marra F, Tacke F. Roles for chemokines in liver disease. Gastroenterology. 2014; 147:577-594. e571. [PubMed: 25066692]

50. Leroux A, Ferrere G, Godie V, et al. Toxic lipids stored by Kupffer cells correlates with their proinflammatory phenotype at an early stage of steatohepatitis. J Hepatol. 2012; 57:141-149. [PubMed: 22425624]

51. Sawada K, Ohtake T, Hasebe T, et al. Augmented hepatic Toll-like receptors by fatty acids trigger the pro-inflammatory state of non-alcoholic fatty liver disease in mice. Hepatology Research. 2014; 44:920-934. [PubMed: 23834389]

52. Fallowfield JA, Mizuno M, Kendall TJ, et al. Scar-associated macrophages are a major source of hepatic matrix metalloproteinase-13 and facilitate the resolution of murine hepatic fibrosis. $\mathbf{J}$ Immunol. 2007; 178:5288-5295. [PubMed: 17404313]

53. Chinetti-Gbaguidi G, Staels B. Macrophage polarization in metabolic disorders: functions and regulation. Curr Opin Lipidol. 2011; 22:365-372. [PubMed: 21825981]

54. Tacke F, Yoneyama H. From NAFLD to NASH to fibrosis to HCC: role of dendritic cell populations in the liver. Hepatology. 2013; 58:494-496. [PubMed: 23519833]

55. Lukacs-Kornek V, Schuppan D. Dendritic cells in liver injury and fibrosis: shortcomings and promises. J Hepatol. 2013; 59:1124-1126. [PubMed: 23727306]

56. Henning JR, Graffeo CS, Rehman A, et al. Dendritic cells limit fibroinflammatory injury in nonalcoholic steatohepatitis in mice. Hepatology. 2013; 58:589-602. [PubMed: 23322710]

57. Sutti S, Locatelli I, Bruzzi S, et al. CX3CR1-expressing inflammatory dendritic cells contribute to the progression of steatohepatitis. Clin Sci (Lond). 2015; 129:797-808. [PubMed: 26253086]

58. Almeda-Valdes P, Aguilar Olivos NE, Barranco-Fragoso B, Uribe M, Mendez-Sanchez N. The Role of Dendritic Cells in Fibrosis Progression in Nonalcoholic Fatty Liver Disease. Biomed Res Int. 2015; 2015:768071. [PubMed: 26339640]

59. Xu R, Huang H, Zhang Z, Wang FS. The role of neutrophils in the development of liver diseases. Cell Mol Immunol. 2014; 11:224-231. [PubMed: 24633014] 
60. Rensen SS, Bieghs V, Xanthoulea S, et al. Neutrophil-derived myeloperoxidase aggravates nonalcoholic steatohepatitis in low-density lipoprotein receptor-deficient mice. PLoS One. 2012; 7:e52411. [PubMed: 23285030]

61. Ibusuki R, Uto H, Arima S, et al. Transgenic expression of human neutrophil peptide-1 enhances hepatic fibrosis in mice fed a choline-deficient, L-amino acid-defined diet. Liver Int. 2013; 33:1549-1556. [PubMed: 23682724]

62. Talukdar S, Ohda Y, Bandyopadhyay G, et al. Neutrophils mediate insulin resistance in mice fed a high-fat diet through secreted elastase. Nat Med. 2012; 18:1407-1412. [PubMed: 22863787]

63. Tian Z, Chen Y, Gao B. Natural killer cells in liver disease. Hepatology. 2013; 57:1654-1662. [PubMed: 23111952]

64. Geissmann F, Cameron TO, Sidobre S, et al. Intravascular immune surveillance by CXCR6+ NKT cells patrolling liver sinusoids. PLoS Biol. 2005; 3:e113. [PubMed: 15799695]

65. Kumar V. NKT-cell subsets: promoters and protectors in inflammatory liver disease. J Hepatol. 2013; 59:618-620. [PubMed: 23669283]

66. Martin-Murphy BV, You Q, Wang H, et al. Mice lacking natural killer T cells are more susceptible to metabolic alterations following high fat diet feeding. PLoS One. 2014; 9:e80949. [PubMed: 24465369]

67. Elinav E, Pappo O, Sklair-Levy M, et al. Adoptive transfer of regulatory NKT lymphocytes ameliorates non-alcoholic steatohepatitis and glucose intolerance in ob/ob mice and is associated with intrahepatic CD8 trapping. J Pathol. 2006; 209:121-128. [PubMed: 16482497]

68. Kremer M, Thomas E, Milton RJ, et al. Kupffer cell and interleukin-12-dependent loss of natural killer T cells in hepatosteatosis. Hepatology. 2010; 51:130-141. [PubMed: 20034047]

69. Tajiri K, Shimizu Y, Tsuneyama K, Sugiyama T. Role of liver-infiltrating CD3+CD56+ natural killer T cells in the pathogenesis of nonalcoholic fatty liver disease. Eur J Gastroenterol Hepatol. 2009; 21:673-680. [PubMed: 19318971]

70. Syn WK, Oo YH, Pereira TA, et al. Accumulation of natural killer T cells in progressive nonalcoholic fatty liver disease. Hepatology. 2010; 51:1998-2007. [PubMed: 20512988]

71. Tajiri K, Shimizu Y. Role of NKT Cells in the Pathogenesis of NAFLD. Int J Hepatol. 2012; 2012:850836. [PubMed: 22577564]

72. Kirpich IA, Marsano LS, McClain CJ. Gut-liver axis, nutrition, and non-alcoholic fatty liver disease. Clin Biochem. 2015; 48:923-930. [PubMed: 26151226]

73. Vajro P, Paolella G, Fasano A. Microbiota and gut-liver axis: their influences on obesity and obesity-related liver disease. J Pediatr Gastroenterol Nutr. 2013; 56:461-468. [PubMed: 23287807]

74. Federico A, Dallio M, Godos J, Loguercio C, Salomone F. Targeting gut-liver axis for the treatment of nonalcoholic steatohepatitis: translational and clinical evidence. Transl Res. Epub ahead of print.

75. Kuipers F, Bloks VW, Groen AK. Beyond intestinal soap--bile acids in metabolic control. Nat Rev Endocrinol. 2014; 10:488-498. [PubMed: 24821328]

76. Liu HX, Keane R, Sheng L, Wan YY. Implications of microbiota and bile acid in liver injury and regeneration. J Hepatol. 2015; 63:1502-10. [PubMed: 26256437]

77. Groschwitz KR, Hogan SP. Intestinal barrier function: molecular regulation and disease pathogenesis. J Allergy Clin Immunol. 2009; 124:3-20. [PubMed: 19560575]

78. Giorgio V, Miele L, Principessa L, et al. Intestinal permeability is increased in children with nonalcoholic fatty liver disease, and correlates with liver disease severity. Dig Liver Dis. 2014; 46:556-560. [PubMed: 24631029]

79. Luther J, Garber JJ, Khalili H, et al. Hepatic Injury in Nonalcoholic Steatohepatitis Contributes to Altered Intestinal Permeability. Cell Mol Gastroenterol Hepatol. 2015; 1:222-232. [PubMed: 26405687]

80. Teixeira TF, Collado MC, Ferreira CL, Bressan J, do Peluzio MC. Potential mechanisms for the emerging link between obesity and increased intestinal permeability. Nutr Res. 2012; 32:637-647. [PubMed: 23084636]

Dig Dis Sci. Author manuscript; available in PMC 2017 May 01. 
81. Miele L, Valenza V, La Torre G, et al. Increased intestinal permeability and tight junction alterations in nonalcoholic fatty liver disease. Hepatology. 2009; 49:1877-1887. [PubMed: 19291785]

82. Schneider KM, Bieghs V, Heymann F, et al. CX3CR1 is a gatekeeper for intestinal barrier integrity in mice: Limiting steatohepatitis by maintaining intestinal homeostasis. Hepatology. 2015; 62:1405-1416. [PubMed: 26178698]

83. Vonghia L, Francque S. Cross talk of the immune system in the adipose tissue and the liver in nonalcoholic steatohepatitis: Pathology and beyond. World J Hepatol. 2015; 7:1905-1912. [PubMed: 26244065]

84. Ramadori P, Kroy D, Streetz KL. Immunoregulation by lipids during the development of nonalcoholic steatohepatitis. Hepatobiliary Surg Nutr. 2015; 4:11-23. [PubMed: 25713801]

85. Moschen AR, Wieser V, Tilg H. Adiponectin: key player in the adipose tissue-liver crosstalk. Curr Med Chem. 2012; 19:5467-5473. [PubMed: 22876924]

86. Wan J, Benkdane M, Teixeira-Clerc F, et al. M2 Kupffer cells promote M1 Kupffer cell apoptosis: a protective mechanism against alcoholic and nonalcoholic fatty liver disease. Hepatology. 2014; 59:130-142. [PubMed: 23832548]

87. Arrese M, Cabrera D, Barrera F. Obeticholic acid: expanding the therapeutic landscape of NASH. Annals of hepatology. 2015; 14:430-432. [PubMed: 25864227]

88. Cariou B, Staels B. GFT505 for the treatment of nonalcoholic steatohepatitis and type 2 diabetes. Expert opinion on investigational drugs. 2014; 23:1441-1448. [PubMed: 25164277] 


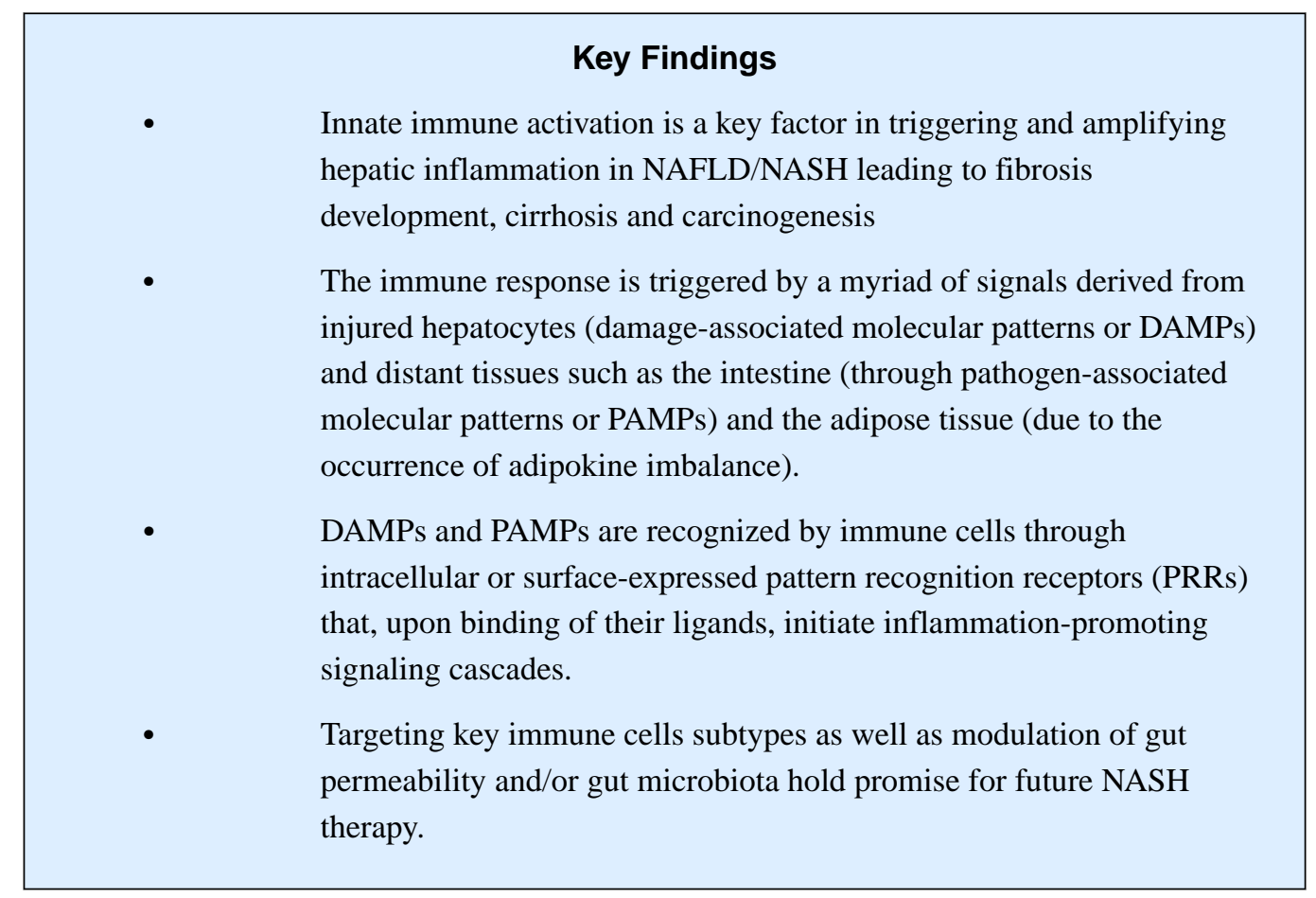




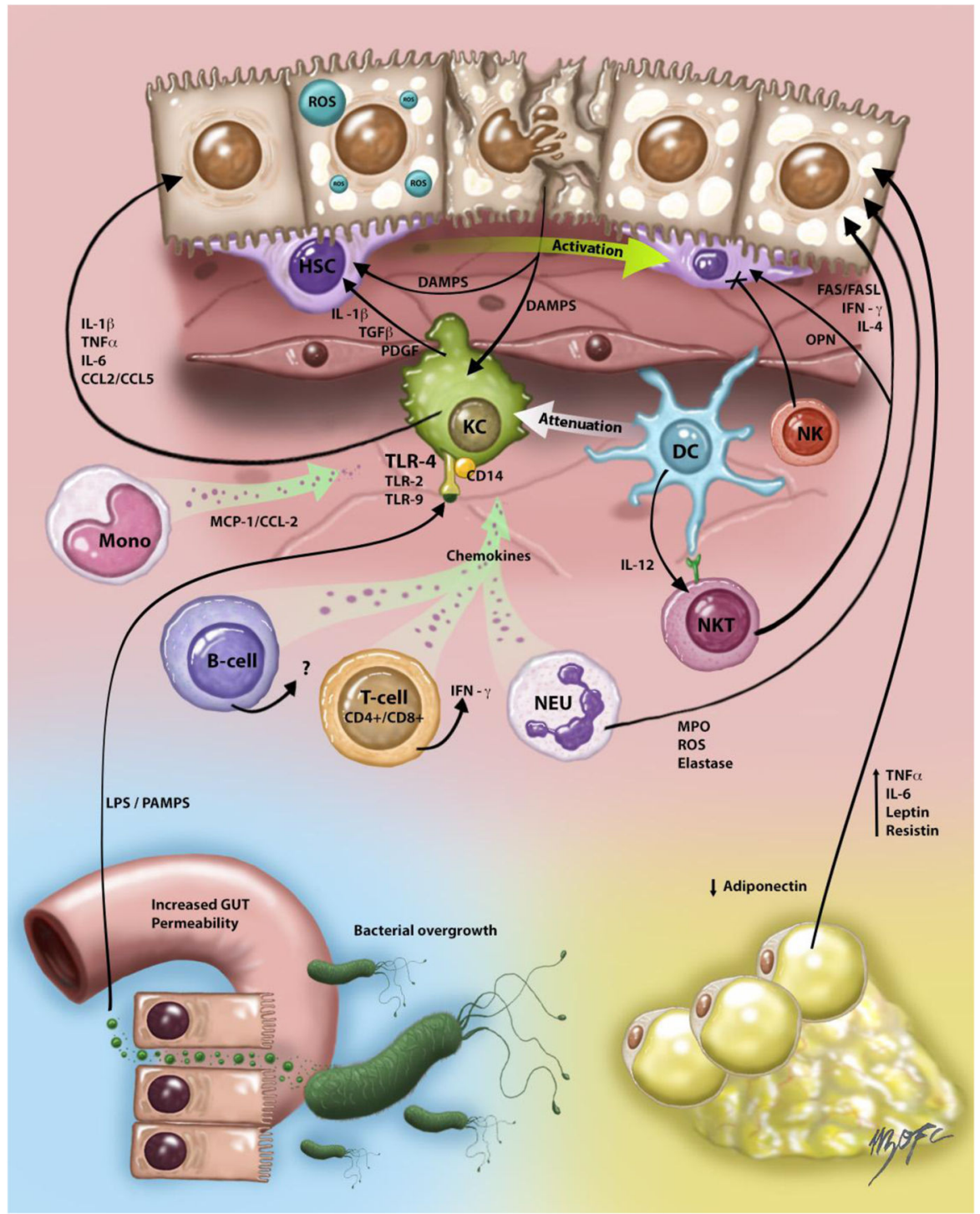

Figure 1. Immune and inflammatory responses in NAFLD/NASH

Innate immune activation is a key factor in triggering and amplifying hepatic inflammation in NAFLD/NASH. Fat overload of hepatocytes induces lipotoxicity and release of damageassociated molecular patterns (DAMPs) that activate both Kupffer cell (KC) and hepatic stellate cells (HSC) promoting inflammation and fibrosis respectively. Activated KCs in turn produce inflammatory cytokines and chemokines such as tumor necrosis factor-alpha (TNFa), Interleukin-1 $\beta$ (IL-1 $\beta$ ), Interleukin-6 (IL-6) and C-C motif ligand 2 and 5 (CCL2 and CCL5) that contribute to injury and inflammatory necrosis in hepatocytes. KC secretion of tumor growth factor- $\beta$ (TGF- $\beta$ ) and platelet-derived growth factor (PDGF) also promotes fibrosis development through the mitogenic stimuli of these factors on HSC. KC population can be replenished by bone marrow-derived monocytes that amplify $\mathrm{KC}$ activation and recruitment through the production of Monocyte chemoattractant protein-1 MCP-1/CCL2. Bacterial-derived products also activate KCs through PAMPs (pathogen-associated molecular patterns), which originate in the intestine and reach the liver via portal circulation 
due to altered intestinal barrier ("leaky gut"). The role of other immune cells types in NAFLD/NASH is less clear. Natural killer (NK) cells function has been found impaired in experimental NASH and these cells could negatively regulate fibrosis through interactions with HSC. Natural killer T (NKT) cells seem to be depleted during steatosis development but increase later in the course of the disease likely contributing to inflammation and fibrosis in NASH through production of interleukin-4 (IL-4), osteopontin (OPN) and interferongamma (IFN- $\gamma$ ). Neutrophils (NEU) exacerbate the ongoing inflammatory state by contributing to macrophage recruitment and cell damage through release of myeloperoxidase, reactive oxygen species (ROS) and elastase. Dendritic cells (DC) DCs also rapidly infiltrate the liver in NASH exhibiting an activated immune phenotype but its depletion exacerbates liver inflammation suggesting that DC attenuates the inflammatory response likely through modulating $\mathrm{KC}$ activation. $\mathrm{B}$ - and $\mathrm{T}$-cell responses also contribute to the feed-forward inflammatory loop through secretion of pro-inflammatory cytokines that stimulates pro-inflammatory KC activation. Finally, dysfunctional adipose tissue disturb the secretion of various adipokines including adiponectin, interleukin-6 (IL-6), leptin, TNF-a and resistin that contribute to inflammation in the liver. Also, since adiponectin is a potent anti-inflammatory and insulin sensitizing adipokine its reduced levels in NAFLD/NASH can also promote hepatic inflammatory responses. 